TRANSACTIONS OF THE

AMERICAN MATHEMATICAL SOCIETY

Volume 356, Number 10, Pages 3881-3897

S 0002-9947(03)03425-1

Article electronically published on December 15, 2003

\title{
THE DELIGNE COMPLEX FOR THE FOUR-STRAND BRAID GROUP
}

\author{
RUTH CHARNEY
}

\begin{abstract}
This paper concerns the homotopy type of hyperplane arrangements associated to infinite Coxeter groups acting as reflection groups on $\mathbb{C}^{n}$. A long-standing conjecture states that the complement of such an arrangement should be aspherical. Some partial results on this conjecture were previously obtained by the author and M. Davis. In this paper, we extend those results to another class of Coxeter groups. The key technical result is that the spherical Deligne complex for the 4-strand braid group is CAT(1).
\end{abstract}

\section{INTRODUCTION}

The motivation for this paper concerns the homotopy type of certain complex hyperplane arrangements, namely those associated to infinite Coxeter groups acting as reflection groups on $\mathbb{C}^{n}$. There is a long-standing conjecture that states that the complement of such an arrangement should be aspherical. Some partial results on this problem were obtained by the author and M. Davis in [5] and by the author and D. Peifer in [7]. Picking up where [5] left off, this paper proves the conjecture for some new classes of infinite Coxeter groups.

We begin by recalling some basic facts about Coxeter groups and Artin groups. A Coxeter group is a group with presentation of the form

$$
W=\left\langle S \mid s^{2}=1,(s t)^{m(s, t)}=1, \forall s, t \in S\right\rangle,
$$

where $S$ is a finite set and $m(s, t)=m(t, s) \in\{2,3, \ldots, \infty\}$. (If $m(s, t)=\infty$, we omit the relation.) The pair $(W, S)$ is called a Coxeter system.

Associated to any Coxeter system is an Artin system $(A, S)$, where $A$ is the group defined by the presentation

$$
A=\langle S \mid \operatorname{prod}(s, t ; m(s, t))=\operatorname{prod}(t, s ; m(s, t)), s, t \in S\rangle,
$$

where $\operatorname{prod}(s, t ; m(s, t))$ denotes the alternating word $s t s t \ldots$ of length $m(s, t)$. Adding the relations $s^{2}=1$ gives back the presentation for $(W, S)$, thus $W$ is a quotient of $A$. Artin groups corresponding to finite Coxeter groups are known as finite type Artin groups.

If $T \subset S$, and $W_{T}$ is the subgroup of $W$ generated by $T$, then $\left(W_{T}, T\right)$ is a Coxeter system. Likewise, if $A_{T}$ denotes the subgroup of $A$ spanned by $T$, then $\left(A_{T}, T\right)$ is the Artin system associated to $\left(W_{T}, T\right)$. The groups $W_{T}$ and $A_{T}$ are

Received by the editors August 6, 2002 and, in revised form, May 1, 2003.

2000 Mathematics Subject Classification. Primary 20F36, 20F55, 52C35.

Key words and phrases. Artin groups, hyperplane arrangements.

This work was partially supported by NSF grant DMS-0104026.

(C)2003 American Mathematical Society 
called special subgroups of $W$ and $A$, respectively, and their conjugates are called parabolic subgroups.

Any finite Coxeter system $(W, S)$ can be realized as a group of linear transformations of $\mathbb{R}^{n}, n=|S|$, with the elements of $S$ acting as orthogonal reflections in the walls of a simplicial cone. For each reflection $r$ in $W(r$ acts as a reflection if it is conjugate to an element of $S$ ), let $H_{r}$ denote the hyperplane fixed by $r$. Then $W$ acts freely on the complement of these hyperplanes. Complexifying the action, we get an action of $W$ on $\mathbb{C}^{n}$ which is free on the complement of the complex hyperplanes $\mathbb{C} H_{r}=H_{r} \oplus i H_{r}$. Let $\mathcal{H}_{W}=\mathbb{C}^{n} \backslash\left(\bigcup_{r} \mathbb{C} H_{r}\right)$ be the hyperplane complement. Deligne [11] showed that $\mathcal{H}_{W} / W$ is aspherical and its fundamental group is the Artin group associated to $W$. In other words, $\mathcal{H}_{W} / W$ is a $K(A, 1)$-space.

More generally, any Coxeter group acts as a reflection group on an $n$-dimensional vector space $V$ with the generators acting as reflections in the walls of a simplicial cone $Z$. In the case of an infinite Coxeter group, the reflections are orthogonal with respect to a certain bilinear form on $V$, but the form is not positive definite. In this case, the picture is not as nice: $Z$ contains points with infinite isotropy group and the translates of $Z$ do not cover $V$. However, Vinberg [18] proved that if we remove the points of infinite isotropy, we get a subspace $Z^{0}$ whose $W$-translates form an open cone $\Omega$ in $V$, known as the Tits cone, and the reflection hyperplanes $\mathbb{C} H_{r}$ form a locally finite arrangement in $\Omega$. We define the hyperplane complement associated to $W$ to be the space

$$
\mathcal{H}_{W}=(\Omega \oplus i \Omega) \backslash\left(\bigcup_{r} \mathbb{C} H_{r}\right) .
$$

For example, if $W$ is an irreducible Euclidean Coxeter group, then the action of $W$ on $V$ preserves an affine hyperplane $\mathbb{A}^{n-1}$. In this case, $Z^{0}$ is $Z-\{0\}$ and $\Omega$ is an open halfspace of $V$ containing $\mathbb{A}$. Thus, there is a linear retraction of $\mathcal{H}_{W}$ onto the complement of a locally finite arrangement of hyperplanes in the complex affine space $\mathbb{A} \oplus i \mathbb{A}$.

We are interested in the following conjecture which is attributed to Arnold, Pham, and Thom.

Conjecture. For an infinite Coxeter system $(W, S)$ with associated Artin group A, the orbit space $\mathcal{H}_{W} / W$ is a $K(A, 1)$-space.

It was shown by van der Lek [17] in the early 1980's that $\pi_{1}\left(\mathcal{H}_{W} / W\right)=A$. Thus, to prove the conjecture, one must show that the universal covering space of the hyperplane complement is contractible. This problem was addressed by the author and M. Davis in 5. In that paper, it was shown that the universal covering space of $\mathcal{H}_{W}$ is homotopy equivalent to a certain simplicial complex $\mathcal{D}_{A}$, known as the Deligne complex of $A$. To show that this simplicial complex is contractible, the authors proposed to find a metric of "nonpositive curvature" on $\mathcal{D}_{A}$. They considered two metrics, a cubical metric and a more natural metric based on a construction of Moussong. The cubical metric was determined to be nonpositively curved if and only if the Coxeter system $(W, T)$ satisfies the "FC condition" (i.e., the condition that $W_{T}$ is finite if and only if $m(s, t)<\infty$ for all $\left.s, t \in T\right)$. The Moussong metric was shown to be nonpositively curved if $\mathcal{D}_{A}$ is 2 -dimensional (i.e., if $W_{T}$ is infinite whenever $|T|>2$ ) and conjectured to be nonpositively curved for all $\mathcal{D}_{A}$. In this paper, we prove that the Moussong metric is nonpositively curved for many of the 3-dimensional, and some higher-dimensional, Deligne complexes (Corollaries 5.5 
and [5.6). The $K(\pi, 1)$ conjecture and a number of algebraic properties for these groups follow immediately.

The author would like to thank the Mathematical Institute at Oxford University for its hospitality during the development of this paper. She would also like to thank John Crisp for helpful conversations.

\section{Coxeter And Deligne complexes}

The notion of "nonpositive curvature" on a geodesic metric space was defined by Gromov in [14] in terms of a "thin triangle" condition. A metric space satisfying this condition globally, is called a $\operatorname{CAT}(0)$ space. We begin with a quick review $\mathrm{CAT}(0)$ spaces. The reader is refered to [1] for more details.

Recall that a piecewise Euclidean complex is a cell complex obtained by gluing together polyhedral Euclidean cells via isometries of faces. Given such a complex, the induced metric is defined by setting $d(x, y)$ equal to the infimum of the lengths of paths from $x$ to $y$. Assuming that there are only finitely many isometry types of simplicies (which will always be the case in this paper), this metric is complete and geodesic, i.e., the infimum is always realized by some path. Such a path is called a geodesic from $x$ to $y$.

A piecewise spherical complex and its induced metric are defined similarly. If $X$ is a piecewise Euclidean complex, then for any vertex $v \in X, \operatorname{link}(v, X)$ is defined as the unit tangent space to $X$ at $v$. The piecewise Euclidean structure on $X$ induces a piecewise spherical structure on $\operatorname{link}(v, X)$ in the obvious manner.

We say that $X$ is $C A T(0)$ if geodesic triangles in $X$ are "at least as thin" as comparison triangles in $\mathbb{R}^{2}$. That is, the distance between any two points on a geodesic triangle $\Delta$ in $X$ is no greater than the distance between the corresponding points on a triangle $\Delta^{\prime}$ of the same side lengths in $\mathbb{R}^{2}$. It is immediate from the definition that geodesics in a $\mathrm{CAT}(0)$ space are unique and vary continuously with their endpoints. It follows that any $C A T(0)$ space is contractible.

To verify that a metric on $X$ is $\operatorname{CAT}(0)$, we must study the induced piecewise spherical metric on the links of vertices. We say a piecewise spherical complex $L$ is CAT(1) if geodesic triangles in $L$ (of perimeter $\leq 2 \pi$ ) are "at least as thin" as comparison triangles in $\mathbb{S}^{2}$. Proofs of the following properties can be found in [1].

Theorem 2.1. (i) A piecewise Euclidean complex $X$ is $C A T(0)$ if and only if $X$ is simply connected and the link of every vertex in $X$ is CAT(1).

(ii) A piecewise spherical complex $L$ is CAT(1) if and only if the link of every vertex in $L$ is $C A T(1)$ and $L$ has no locally geodesic loops of length less than $2 \pi$.

Now let $(W, S)$ be a Coxeter system and let $A$ be the associated Artin group. As discussed in the Introduction, the hyperplane complement conjecture reduces (by the results of [5]) to proving that the Deligne complex, $\mathcal{D}_{A}$, is contractible. The Deligne complex is modelled on the Davis-Coxeter complex, $\mathcal{C}_{W}$, which was first introduced by Davis in [10] and shown by Moussong in [15] to carry a CAT(0) metric. The remainder of this section is devoted to the construction of these two complexes and a description of the Moussong metric.

We begin with some notation. Let

$$
\begin{aligned}
\mathcal{S}^{f} & =\left\{T \mid T \subseteq S, W_{T} \text { is finite }\right\}, \\
W \mathcal{S}^{f} & =\left\{w W_{T} \mid w \in W, T \in \mathcal{S}^{f}\right\} .
\end{aligned}
$$


Here, we include $T=\emptyset$ with the convention that $W_{\emptyset}=\{1\}$. These sets are partially ordered by inclusion. The Davis-Coxeter complex for $W$, which we denote by $\mathcal{C}_{W}$, is the flag complex associated to the poset $W \mathcal{S}^{f}$. That is, $\mathcal{C}_{W}$ is the simplicial complex whose vertices are the elements of $W \mathcal{S}^{f}$ and whose $k$-simplices are totally ordered subsets (or "flags") of length $k+1$. (This complex is a modification of the classical Coxeter complex defined by Tits. Tits' construction does not require that $W_{T}$ be finite.) $W$ acts by left multiplication on $\mathcal{C}_{W}$ and a fundamental domain for this action is the subcomplex $K$ spanned by the vertices $W_{T}$. Note that this subcomplex is isomorphic to the flag complex associated to $\mathcal{S}^{f}$.

Now suppose $W$ is finite. Then $\mathcal{S}^{f}$ is the set of all subsets of $S$ and hence $K$ can be combinatorially identified with a cube of dimension $n=|S|$. (To see the cubical structure, ignore all edges except those corresponding to inclusions $W_{T} \subset W_{T^{\prime}}$ with $\left|T^{\prime}\right|=|T|+1$.) Moreover, $W \mathcal{S}^{f}$ has a unique maximal element $W=W_{S}$, so $\mathcal{C}_{W}$ is a cone.

For finite $W, \mathcal{C}_{W}$ can be given the metric of a convex polyhedral cell as follows. Realize $W$ as a group of orthogonal transformations of $\mathbb{R}^{n}$ with the generators $S$ acting as reflections in the walls of a simplicial cone $Z$. There is a unique point $x_{\emptyset}$ in the interior of $Z$ whose distance from every wall is 1 . Let $X \subset \mathbb{R}^{n}$ be the convex hull of the orbit $W x_{\emptyset}$. Then $X$ is equivariantly isomorphic to $\mathcal{C}_{W}$ with the origin corresponding to the cone point in $K, x_{\emptyset}$ corresponding to $W_{\emptyset}$, and $K$ identified with $Z \cap X$. The complex $\mathcal{C}_{W}$, with this metric, is known as the Coxeter cell for $W$.

Returning to the case of a (possibly) infinite $W$, Moussong [15] defined a piecewise Euclidean metric on the Davis-Coxeter complex as follows. We can cover $\mathcal{C}_{W}$ by the closed stars of the maximal vertices $w W_{T}$ (maximal with respect to the partial order on $W \mathcal{S}^{f}$ ). This star can be naturally identified with the Davis-Coxeter complex for $W_{T}$ (translated by $w$ ). Since $W_{T}$ is finite, we can assign this star the metric of the Coxeter cell $\mathcal{C}_{W_{T}}$. These cells fit together to give a piecewise Euclidean structure on $\mathcal{C}_{W}$. Moussong proved that the induced metric is $\operatorname{CAT}(0)$ for all $W$.

The Deligne complex for the Artin group $A$ is defined analogously. Let

$$
A \mathcal{S}^{f}=\left\{a A_{T} \mid a \in A, T \in \mathcal{S}^{f}\right\}
$$

partially ordered by inclusion. Then the Deligne complex, $\mathcal{D}_{A}$, is the flag complex associated to this poset. There is a left action of $A$ on $\mathcal{D}_{A}$ and the fundamental domain for this action is again $K$ (viewed as the subcomplex spanned by the vertices $\left.A_{T}\right)$. The quotient map $A \rightarrow W$ induces an equivariant projection of $\mathcal{D}_{A} \rightarrow \mathcal{C}_{W}$ which is an isomorphism on $K$. We define a piecewise Euclidean structure on $\mathcal{D}_{A}$ by declaring each translate of $K$ to be isometric to its image in $\mathcal{C}_{W}$. The induced metric $d$ on $\mathcal{D}_{A}$ is called the Moussong metric.

To prove that the Moussong metric on $\mathcal{D}_{A}$ is $\operatorname{CAT}(0)$, we need to look at the links of vertices in $\mathcal{D}_{A}$. First consider the case in which $W$ is finite. The link of the cone point in the Coxeter cell $\mathcal{C}_{S}$ is an $(n-1)$-sphere with the standard, round metric. Simplicially (with respect to the cubical structure on $\mathcal{C}_{S}$ ), this link has one top-dimensional simplex (or "chamber") for each element of $W$ and one $k$-simplex for each coset $w W_{T}$ with $|T|=n-1-k$. This simplicial decomposition of the sphere is called the spherical Coxeter complex for $W$. We denote it by $\mathcal{C}_{W}^{0}$.

Similarly, in the case where $W$ is finite (so $A$ is finite type), $\mathcal{D}_{A}$ is a cone with cone point $A=A_{S}$. The link of the cone point, with the induced piecewise spherical metric, is the spherical Deligne complex for $A$ which we denote by $\mathcal{D}_{A}^{0}$. It has one 
$k$-simplex for each coset $a A_{T}$ with $|T|=n-1-k$. Note that $\mathcal{C}_{W}^{0}$ and $\mathcal{D}_{A}^{0}$ are defined only for finite Coxeter groups $W$.

Now consider the link of a vertex in $\mathcal{D}_{A}$ for an infinite type Artin group $A$. Any vertex of $\mathcal{D}_{A}$ is the translate of some vertex $v=A_{T}$ in $K$. The star of $v$ is spanned by two types of vertices: those greater than $A_{T}$ (with respect to the partial order on $\left.A \mathcal{S}^{f}\right)$ and those less than $A_{T}$. This gives rise to a decomposition of $\operatorname{link}\left(v, \mathcal{D}_{A}\right)$ as an orthogonal join as described in the following lemma. (For a proof of this lemma see [2], Lemma 2.2.)

Lemma 2.2. Let $T \in \mathcal{S}^{f}$ and let $v=A_{T}$. Let $K_{\geq T}$ be the subcomplex of $K$ spanned by $\left\{A_{R} \mid R \geq T, R \in \mathcal{S}^{f}\right\}$. Then link $\left(v, \mathcal{D}_{A}\right)$ is isometric to the orthogonal join

$$
\operatorname{link}\left(v, \mathcal{D}_{A}\right)=\operatorname{link}\left(v, K_{\geq T}\right) * \mathcal{D}_{A_{T}}^{0} .
$$

Proposition 2.3. The Moussong metric on the Deligne complex $\mathcal{D}_{A}$ is $C A T(0)$ if and only if the spherical Deligne complex $\mathcal{D}_{A_{T}}^{0}$ is CAT(1) for every $T \in \mathcal{S}^{f}$.

Proof. In [5] it is shown that $\mathcal{D}_{A}$ is simply connected, thus by Theorem 2.1] it suffices to prove that the link of every vertex is CAT(1). It was shown by Moussong [15] that $\operatorname{link}\left(A_{T}, K_{\geq T}\right)$ is $\mathrm{CAT}(1)$ for all $T \in \mathcal{S}^{f}$. By [5] (appendix), the join of two CAT(1) spaces is CAT(1), so the proposition follows from Lemma 2.2

Thus, to prove the hyperplane complement conjecture, it would suffice to show that the spherical Deligne complex for every finite type Artin group is CAT(1). It seems that a good place to start is with the Artin groups of small type, that is, Artin groups for which all $m(s, t) \in\{2,3\}$. Crisp [8] has shown that every finite type Artin group $A$ injects into a finite type Artin group $A^{\text {small }}$ of small type. These homomorphisms induce equivariant maps between the corresponding Deligne complexes.

Proposition 2.4. Suppose that the spherical Deligne complex for every small, finite type Artin group is CAT(1). Then the same holds for every finite type Artin group containing no irreducible factor of type $\mathrm{H}_{3}$ or $\mathrm{H}_{4}$ (where $\mathrm{H}_{i}$ refers to the standard Dynkin diagrams for finite irreducible Coxeter groups).

Proof. Suppose $A$ is an irreducible, finite type Artin group. For $A$ of rank 2, the spherical Deligne complex was shown to be CAT(1) in [5]. For rank $\geq 3$, if $A$ is not of type $H_{3}$ or $H_{4}$, then $A$ can be realized as the fixed set of an involution of some small, finite type Artin group. Thus, the proposition follows immediately from a theorem of Crisp, 9], Theorem 23.

The basis of Crisp's argument is that the fixed set of a finite group action on a $\mathrm{CAT}(0)$ space is a convex subset, so the inclusion of $\mathcal{D}_{A}$ into $\mathcal{D}_{A^{\text {small }}}$ is an isometric embedding. We conjecture that for $A$ of type $H_{3}$ and $H_{4}$, the inclusions into small type Artin groups (of type $D_{6}$ and $E_{8}$ ) also induce isometric embeddings on Deligne complexes, but as these groups do no arise as fixed point sets, a different method of proof will be required.

In this paper we consider the first interesting case of a spherical Deligne complex for an Artin group of small type, namely, the two-dimensional complex associated to the braid group on four strands. (This group is the only rank 3 Artin group which is irreducible, small, and of finite type.) Deligne complexes are "buildinglike" in the sense that they can be covered by Davis-Coxeter complexes and links of vertices are lower-dimensional (spherical) Deligne complexes. We hope that some 
inductive argument, along the lines of [6], Theorem 4.1, will allow us to extend these results to higher rank Artin groups of small type in the future. Our experience with spherical buildings suggests that the 2-dimensional case is special and any induction argument will have to start at dimension three.

\section{The CoXeter COMPleX For the Symmetric Group}

We now specialize to the case where $W=S y m_{4}$ is the symmetric group on four letters and the associated Artin group $A=B_{4}$ is the four-strand braid group. In this section we describe an explicit model for the spherical Coxeter complex $\mathcal{C}^{0}=\mathcal{C}_{W}^{0}$ and the spherical Deligne complex $\mathcal{D}^{0}=\mathcal{D}_{A}^{0}$.

Let $V$ be the 3-dimensional vector space

$$
V=\left\{\left(x_{1}, x_{2}, x_{3}, x_{4}\right) \in \mathbb{R}^{4} \mid \sum x_{i}=0\right\} .
$$

Then $W$ acts on $V$ by permuting the coordinates. A transposition $(i, j)$ in $W$ fixes the hyperplane $H_{i, j} \subset V$ defined by $x_{i}=x_{j}$. Consider the unit sphere $\mathbb{S}(V)=\mathbb{S}^{4} \cap V$. The six hyperplanes $H_{i, j}$ divide $\mathbb{S}(V)$ into simplices. This simplicial decomposition of the 2 -sphere is a realization of the spherical Coxeter complex for $W$. From now on, we identify $\mathcal{C}^{0}$ with this realization.

The 2-dimensional simplices of $\mathcal{C}^{0}$ are called chambers. $W$ acts freely, transitively on the set of chambers, thus all chambers are isometric to each other. Consider the chamber defined by $x_{1} \geq x_{2} \geq x_{3} \geq x_{4}$. We call this the fundamental chamber and denote it by $K^{0}$. The sides of this chamber are formed by the hyperplanes $H_{1,2}$, $H_{2,3}$, and $H_{3,4}$.

The standard Coxeter presentation for $W$ is

$$
W=\left\langle r, s, t \mid r^{2}=s^{2}=t^{2}=1,(r t)^{2}=(r s)^{3}=(s t)^{3}=1\right\rangle,
$$

where $r, s, t$ are the transpositions

$$
r=(1,2) \quad s=(2,3) \quad t=(3,4) .
$$

With this notation, the sides of the triangle $K^{0}$ are formed by the intersection of $\mathbb{S}(V)$ with the hyperplanes $H_{r}, H_{s}$, and $H_{t}$ and we refer to them as edges of type $r, s$, or $t$, respectively. The vertex between the edges of type $r$ and $s$ will be called a vertex of type $\{r, s\}$ and similarly for vertices of type $\{r, t\}$ and $\{s, t\}$. Thus, a vertex or edge of type $T \subset\{r, s, t\}$ is fixed by the subgroup $W_{T}$. For any other chamber $w K^{0}$, we define the type of the edges and vertices of $w K^{0}$ similarly, so that the vertex or edge of type $T$ is fixed by the parabolic subgroup $w W_{T} w^{-1}$.

Lemma 3.1. The chambers of $\mathcal{C}^{0}$ satisfy the following:

(1) The angle at a vertex of type $\{r, s\}$ or $\{s, t\}$ is $\pi / 3$ and the angle at a vertex of type $\{r, t\}$ is $\pi / 2$.

(2) The length of an edge of type $r$ or $t$ is $\alpha=\arccos \left(\frac{1}{\sqrt{3}}\right)$ and the length of an edge of type $s$ is $\beta=\arccos \left(\frac{1}{3}\right)$.

(3) These lengths satisfy $2 \alpha>\beta>\alpha$ and $2 \alpha+\beta=\pi$.

Proof. The normal vectors to $H_{r}, H_{s}, H_{t}$ are

$$
\mathbf{n}_{r}=\frac{1}{\sqrt{2}}(1,-1,0,0), \quad \mathbf{n}_{s}=\frac{1}{\sqrt{2}}(0,1,-1,0), \quad \mathbf{n}_{t}=\frac{1}{\sqrt{2}}(0,0,1,-1) .
$$

The lemma now follows by an exercise in trigonometry which we leave to the reader. 


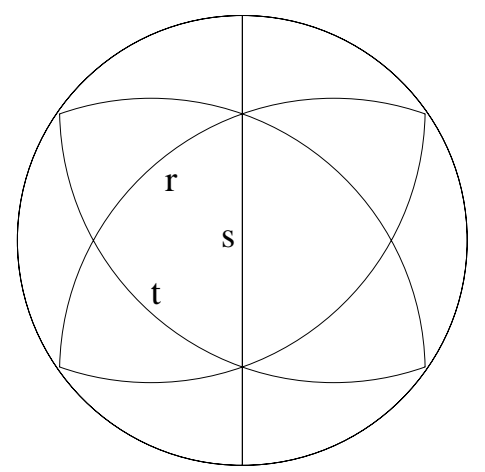

Figure 1. The spherical Coxeter complex for $\mathrm{Sym}_{4}$

The Artin group $A$ associated to $S y m_{4}$ is the 4 -strand braid group $B_{4}$ given by the presentaion

$$
\left.A=B_{4}=\langle r, s, t| r s r=s r s, \text { sts }=t s t, r t=t r\right\rangle .
$$

(In standard notation, $r=\sigma_{1}, s=\sigma_{2}, t=\sigma_{3}$, where $\sigma_{i}$ crosses the $i^{\text {th }}$ string over the $(i+1)^{s t}$ string.) Let $\mathcal{D}^{0}=\mathcal{D}_{A}^{0}$ be the spherical Deligne complex for $A$. Then a fundamental domain for the action of $A$ on $\mathcal{D}^{0}$ is the 2-simplex with vertices $A_{r, s}, A_{r, t}, A_{s, t}$. Thus, we can define the "type" of a vertex or edge analogously. Taking the metric on each 2-simplex of $\mathcal{D}^{0}$ to be the same as the metric on $K^{0}$, we see that Lemma 3.1 also applies to chambers of $\mathcal{D}^{0}$.

\section{RELATIONS IN THE FOUR-STRAND BRAID GROUP}

In this section we prove two key technical lemmas about relations in the fourstrand braid group which will be needed in the proof of the main theorem. Throughout this section $(A, S)=\left(B_{4},\{r, s, t\}\right)$.

Lemma 4.1. Let $T=\{s, t\}$ and $R=\{r, s\}$. Suppose $\tau_{1}, \tau_{2} \in A_{T}$ and $\rho_{1}, \rho_{2} \in A_{R}$ are such that $\tau_{1} \rho_{1}=\rho_{2} \tau_{2}$, and assume that no $\tau_{i}$ or $\rho_{i}$ lies in $A_{s}$. Then there exists $i, j, k_{1}, k_{2}, k_{3}, k_{4} \in \mathbb{Z}$ such that

$$
\begin{array}{ll}
\tau_{1}=s^{k_{1}} t^{j} s^{k_{2}}, & \rho_{1}=s^{-k_{2}} r^{i} s^{k_{4}}, \\
\rho_{2}=s^{k_{1}} r^{i} s^{k_{3}}, & \tau_{2}=s^{-k_{3}} t^{j} s^{k_{4}} .
\end{array}
$$

Proof. We identify the four strand braid group $A$ with the mapping class group of the disk with four punctures, $\Sigma=D^{2}-\left\{x_{1}, x_{2}, x_{3}, x_{4}\right\}$, with $r$ interchanging $x_{1}, x_{2}, s$ interchanging $x_{2}, x_{3}$, and $t$ interchanging $x_{3}, x_{4}$ (see Figure 41). Let $\gamma_{r}, \gamma_{t}$ be the paths shown in Figure 2. Let $C_{r}, C_{s}, C_{t}$ denote the closures of the three components of $\Sigma-\left(\gamma_{t} \cup \gamma_{s}\right)$ as indicated in the figure.

Set $a=\tau_{1} \rho_{1}=\rho_{2} \tau_{2}$, and represent $a$ by a homeomorphism $h: \Sigma \rightarrow \Sigma$ chosen as follows. First choose a representatives $h_{\tau_{1}}$ and $h_{\rho_{1}}$ of $\tau_{1}$ and $\rho_{1}$ satisfying

(1) $\left.h_{\tau_{1}}\right|_{C_{r}}=i d_{C_{r}}$,

(2) $\left.h_{\rho_{1}}\right|_{C_{t}}=i d_{C_{t}}$, and

(3) $h_{\tau_{1}}\left(\gamma_{t}\right)$ has minimal intersection with $\gamma_{t}$. 


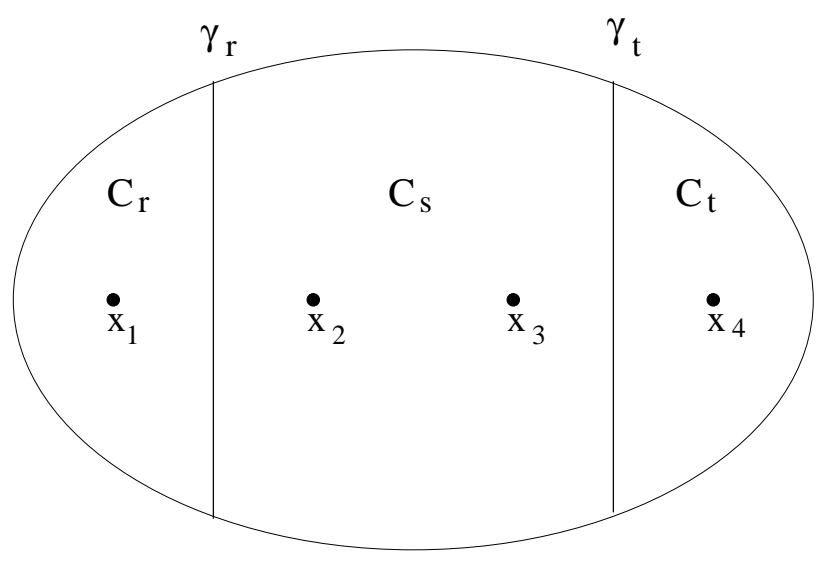

FIGURE 2. The punctured disk $\Sigma$

Now set $h=h_{\tau_{1}} \circ h_{\rho_{1}}$. Consider the action of $h$ on $\gamma_{t}$. Since $\gamma_{t} \cap \gamma_{r}=\emptyset$, we have

(1) $h\left(\gamma_{t}\right) \cap h\left(\gamma_{r}\right)=\emptyset$, and

(2) $h\left(\gamma_{t}\right) \cap \gamma_{r}=h_{\tau_{1}}\left(\gamma_{t}\right) \cap h_{\tau_{1}}\left(\gamma_{r}\right)=\emptyset$.

On the other hand, if we choose representatives $h_{\rho_{2}}$ and $h_{\tau_{2}}$ for $\rho_{2}$ and $\tau_{2}$ in a similar manner, we get a homeomorphism $h^{\prime}=h_{\rho_{2}} \circ h_{\tau_{2}}$, representing $a$, which has the property that $h^{\prime}\left(\gamma_{r}\right) \cap \gamma_{t}=\emptyset$. Since $h^{\prime}$ is isotopic to $h, h\left(\gamma_{r}\right)$ is homotopic to a path which is disjoint from $\gamma_{t}$.

We claim that we can find a path $\beta \sim h\left(\gamma_{r}\right)$ which is simultaneously disjoint from $\gamma_{t}$ and $h\left(\gamma_{t}\right)$. To see this, set $\beta_{0}=h\left(\gamma_{r}\right)$, so $\beta_{0}$ is disjoint from $h\left(\gamma_{t}\right)$ but may intersect $\gamma_{t}$ in, say, $n$ points. We may assume (by a local deformation of $h_{\tau_{1}}$ ) that these intersections are transverse. Consider the complement of $\gamma_{t} \cup \beta_{0}$ in $\Sigma$. Since $\beta_{0}$ can be homotoped off of $\gamma_{t}$, some component $D$ of this complement must be a disk. This disk cannot contain any segment of $h\left(\gamma_{t}\right)$ since this would contradict the assumption that $h_{\tau_{1}}\left(\gamma_{t}\right)\left(=h\left(\gamma_{t}\right)\right)$ has minimal intersection with $\gamma_{t}$. It follows that $\beta_{0}$ can be homotoped across $D$ to a path $\beta_{1}$ which is still disjoint from $h\left(\gamma_{t}\right)$, but intersects $\gamma_{t}$ in $n-2$ points. Repeat this process until no intersections remain. This proves the claim.

We now have a pair of disjoint, simple closed curves, $\beta$ and $h\left(\gamma_{t}\right)$, such that $\beta \subset \Sigma-C_{t}$ and $h\left(\gamma_{t}\right) \subset \Sigma-C_{r}$. Consider the segments $\left\{\alpha_{i}\right\}$ of $\beta$ which lie in the twice-punctured disk $C_{s}$. If some $\alpha_{i}$ is homotopic to a segment of $\gamma_{r}$, we slide it back into $C_{r}$ and ignore it. The remaining segments are mutually disjoint, nontrivial curves in $C_{s}$, hence there is an element of $A_{s}$, the mapping class group of $C_{s}$, which maps every $\alpha_{i}$ to a curve parallel to one of the three curves $\sigma_{1}, \sigma_{2}, \sigma_{3}$ in Figure 3 Say $s^{k} \in A_{s}$ is such an element.

Note that $s^{k}(\beta)$ and $s^{k} h\left(\gamma_{t}\right)$ are still disjoint, simple curves lying in $\Sigma-C_{t}$ and $\Sigma-C_{r}$, respectively. Suppose that $s^{k}(\beta)$ contains some segment of type $\sigma_{3}$, or contains segments of both types $\sigma_{1}$ and $\sigma_{2}$. In this case, $s^{k} h\left(\gamma_{t}\right)$ lies in a disk with only one puncture, namely $x_{4}$. Since $s^{k} h\left(\gamma_{t}\right)$ cannot be homotopic to the boundary of $\Sigma$, the only possibility is that $s^{k} h\left(\gamma_{t}\right) \sim \gamma_{t}$. It follows that, up to homotopy, $s^{k} h_{\tau_{1}}$ fixes both $\gamma_{r}$ and $\gamma_{t}$. Thus $s^{k} \tau_{1}$ lies in $A_{s}$, the mapping class group of $C_{s}$. But this contradicts our hypothesis that $\tau_{i} \notin A_{s}$. 


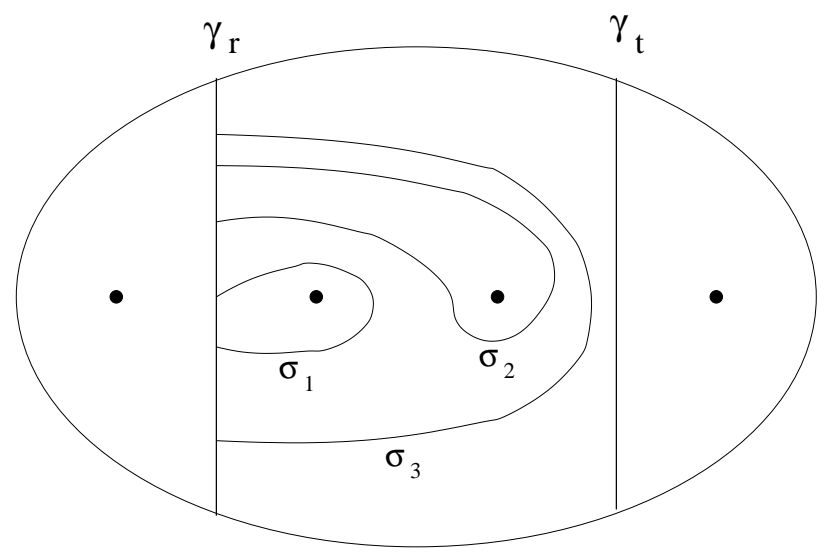

Figure 3. The curves $\sigma_{1}, \sigma_{2}, \sigma_{3}$

Thus, all segments of $s^{k}(\beta)$ between $\gamma_{r}$ and $\gamma_{t}$ are of type $\sigma_{1}$ or all are of type $\sigma_{2}$. Replacing $s^{k}$ by $s^{k+1}$ if necessary, we may assume that they are all of type $\sigma_{1}$. In this case, $s^{k} h\left(\gamma_{t}\right)$ lies in a disk with two punctures, $x_{3}, x_{4}$, whose mapping class group is $A_{t}$, so $s^{k} h\left(\gamma_{t}\right) \sim t^{j}\left(\gamma_{t}\right)$ for some $j$. It follows that $t^{-j} s^{k} h_{\tau_{1}}$ fixes both $\gamma_{r}$ and $\gamma_{t}$ up to homotopy. Thus, $t^{-j} s^{k} \tau_{1}=s^{k_{2}}$ for some $k_{2}$, so $\tau_{1}=s^{-k} t^{j} s^{k_{2}}$. Likewise, $s^{k}(\beta)$ is contained in a disk with two punctures, $x_{1}, x_{2}$ and, since $\beta \sim h\left(\gamma_{r}\right) \sim h_{\rho_{2}}\left(\gamma_{r}\right)$, a similar argument shows that $\rho_{2}=s^{-k} r^{i} s^{k_{3}}$ for some $i, k_{3}$.

Set $k_{1}=-k$. To complete the lemma, note that

$$
\begin{aligned}
\tau_{1} \rho_{1} & =\rho_{2} \tau_{2} \\
\Longrightarrow s^{k_{1}} t^{j} s^{k_{2}} \rho_{1} & =s^{k_{1}} r^{i} s^{k_{3}} \tau_{2} \\
\Longrightarrow \quad r^{-i} s^{k_{2}} \rho_{1} & =t^{-j} s^{k_{3}} \tau_{2} .
\end{aligned}
$$

The left-hand side of the last equality lies in $A_{R}$ while the right-hand side lies in $A_{T}$, hence both must lie in $A_{R} \cap A_{T}=A_{s}$. We conclude that, for some $k_{4} \in \mathbb{Z}$, $\rho_{1}=s^{-k_{2}} r^{i} s^{k_{4}}$ and $\tau_{2}=s^{-k_{3}} t^{j} s^{k_{4}}$.

Lemma 4.2. Let $R=\{r, s\}$ and let $\rho_{1}, \rho_{2}, \rho_{3} \in A_{R}$. Suppose that for some $i_{1}, i_{2}, i_{3} \neq 0$,

$$
t^{i_{1}} \rho_{1} t^{i_{2}} \rho_{2} t^{i_{3}} \rho_{3}=1
$$

Then for $j=1,2,3$, there exist $k_{j}$ such that $\rho_{j} \in A_{r} s^{k_{j}} A_{r}$.

Proof. We first show that it suffices to prove that one of the $\rho$ 's is of the form $r^{i} s^{k} r^{j}$. Since the relation (1) can be cyclically permuted by conjugation, we may suppose without loss of generality, that $\rho_{2}=r^{i} s^{k} r^{j}$. In this case we have

$$
\begin{aligned}
t^{i_{1}} \rho_{1} t^{i_{2}} r^{i} s^{k} r^{j} t^{i_{3}} \rho_{3} & =1, \\
\left(t^{i_{1}}\right)\left(\rho_{1} r^{i}\right)\left(t^{i_{2}} s^{k} t^{i_{3}}\right)\left(r^{j} \rho_{3}\right) & =1, \\
\left(t^{i_{2}} s^{k} t^{i_{3}}\right)\left(r^{j} \rho_{3}\right) & =\left(r^{-i} \rho_{1}^{-1}\right)\left(t^{-i_{1}}\right) .
\end{aligned}
$$

Applying Lemma 4.1 with $\tau_{2}=t^{-i_{1}}$, gives the desired form for $\rho_{1}$ and $\rho_{3}$.

We now prove that some $\rho_{j}$ lies in $A_{r} s^{k_{j}} A_{r}$. Choose a basepoint $x_{0}$ which lies on the boundary of $\Sigma$. The fundamental group $F=\pi_{1}\left(\Sigma, x_{0}\right)$ is a free group on four generators, $q_{1}, q_{2}, q_{3}, q_{4}$, where $q_{i}$ is the loop which winds once around $x_{i}$ counterclockwise. Identifying the braid group $A$ with the mapping class group of $\Sigma$ gives 


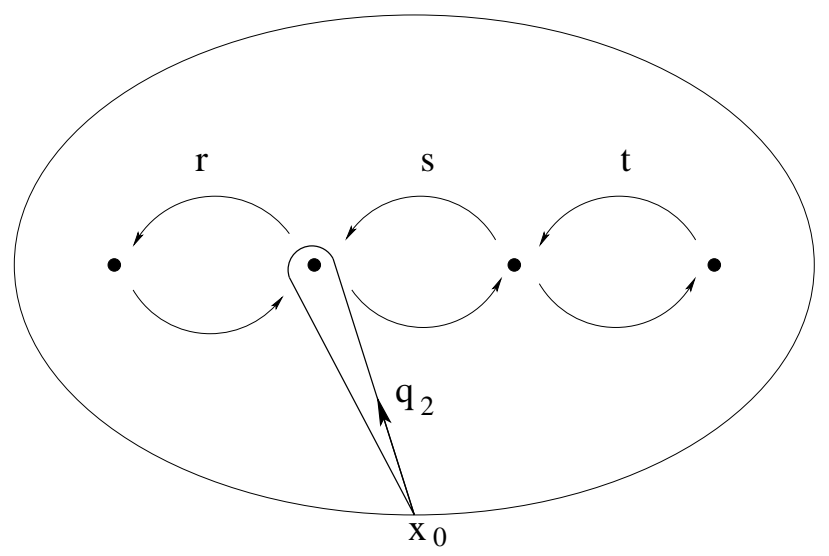

Figure 4 . The action of $r, s$, and $t$ on $q_{2}$

an injection from $A$ into the automorphism group of $F$. Explicitly, the action is given by

$$
\begin{array}{lll}
r\left(q_{1}\right)=q_{2}, & s\left(q_{1}\right)=q_{1}, & t\left(q_{1}\right)=q_{1}, \\
r\left(q_{2}\right)=q_{2} q_{1} q_{2}^{-1}, & s\left(q_{2}\right)=q_{3}, & t\left(q_{2}\right)=q_{2}, \\
r\left(q_{3}\right)=q_{3}, & s\left(q_{3}\right)=q_{3} q_{2} q_{3}^{-1}, & t\left(q_{3}\right)=q_{4}, \\
r\left(q_{4}\right)=q_{4}, & s\left(q_{4}\right)=q_{4}, & t\left(q_{4}\right)=q_{4} q_{3} q_{4}^{-1} .
\end{array}
$$

Note, in particular, that for $k>0$

$$
\begin{aligned}
t^{k}\left(q_{3}\right) & =(\underbrace{q_{4} q_{3} q_{4} \ldots}_{k})(\underbrace{\ldots q_{4}^{-1} q_{3}^{-1} q_{4}^{-1}}_{k-1}), \\
t^{-k}\left(q_{3}\right) & =(\underbrace{q_{3}^{-1} q_{4}^{-1} q_{3}^{-1} \ldots}_{k})(\underbrace{\ldots q_{3} q_{4} q_{3}}_{k+1}) .
\end{aligned}
$$

First assume that $\left|i_{j}\right|>1$ for some $j \in\{1,2,3\}$. By cyclic permutation of the relation (1), we may assume that $\left|i_{3}\right|>1$. Let

$$
a=t^{i_{1}} \rho_{1} t^{i_{2}}=\left(\rho_{2} t^{i_{3}} \rho_{3}\right)^{-1}
$$

and consider the action of $a$ on $q_{1}$. We have $a\left(q_{1}\right)=t^{i_{1}} \rho_{1}\left(q_{1}\right)=t^{i_{1}}(w)$, where $w$ is a word in $q_{1}, q_{2}, q_{3}$. Thus, $a\left(q_{1}\right) \in\left\langle q_{1}, q_{2}, t^{i_{1}}\left(q_{3}\right)\right\rangle$, the subgroup of $F$ generated by $q_{1}, q_{2}, t^{i_{1}}\left(q_{3}\right)$. In particular, any two consecutive occurrences of $q_{4}$ (or power of $q_{4}$ ) in the reduced form of $a\left(q_{1}\right)$ are separated by one of the following:

(1) a power of $q_{3}$,

(2) (if $i_{1}>0$ ) a word in $\left\langle q_{1}, q_{2}\right\rangle$,

(3) (if $i_{1}<0$ ) a word in $q_{3}\left\langle q_{1}, q_{2}\right\rangle q_{3}^{-1}$.

On the other hand, $a\left(q_{1}\right)=\rho_{3}^{-1} t^{-i_{3}} \rho_{2}^{-1}\left(q_{1}\right)=\rho_{3}^{-1}(u)$, where $u \in\left\langle q_{1}, q_{2}, t^{-i_{3}}\left(q_{3}\right)\right\rangle$. Since $\left|i_{3}\right|>1$, the reduced form of $u$ contains two copies of $q_{4}^{ \pm 1}$ separated by a power of $q_{3}$. Since $\rho_{3}^{-1}$ fixes $q_{4}$ and preserves the subgroup generated by $q_{1}, q_{2}, q_{3}$, it follows that the reduced form of $a\left(q_{1}\right)$ contains two copies of $q_{4}^{ \pm 1}$ separated by a power of $\rho_{3}^{-1}\left(q_{3}\right)$. Comparing this with the previous paragraph, we see that $\rho_{3}^{-1}\left(q_{3}\right)$ must be one of the three types of words listed above.

Moreover, $\rho_{3}^{-1}\left(q_{3}\right)$ can be represented by a simple closed curve in $\Sigma$, oriented counter-clockwise, which encloses a single puncture. The only words in $\left\langle q_{1}, q_{2}\right\rangle$ 
with this property are words of the form $r^{j}\left(q_{2}\right)$ (since the mapping class group of $D^{2}-\left\{x_{1}, x_{2}\right\}$ is just $\left.A_{r}\right)$. It follows that the three cases above give, respectively,

(1) $\rho_{3}^{-1}\left(q_{3}\right)=q_{3}$,

(2) $\rho_{3}^{-1}\left(q_{3}\right)=r^{j}\left(q_{2}\right)=r^{j} s^{-1}\left(q_{3}\right)$,

(3) $\rho_{3}^{-1}\left(q_{3}\right)=q_{3} r^{j}\left(q_{2}\right) q_{3}^{-1}=r^{j} s\left(q_{3}\right)$.

In the first case, it follows that $\rho_{3}$ fixes both $q_{3}$ and $q_{4}$ and hence is supported on a disk $\Sigma^{\prime} \subset \Sigma$ with only two punctures, $x_{1}, x_{2}$. Thus $\rho_{3}$ lies in the mapping class group $A_{r}$ of $\Sigma^{\prime}$. In the other two cases, the same reasoning applies to $\rho_{3} r^{j} s^{ \pm}$to give $\rho_{3} r^{j} s^{ \pm}=r^{k}$ for some $k$.

It remains to consider the case where $\left|i_{1}\right|=\left|i_{2}\right|=\left|i_{3}\right|=1$. Say all three of the $i_{j}$ have the same sign. Inverting the relation (1) if necessary, we may assume that all $i_{j}$ are positive. Let $a$ be as above and consider $a\left(q_{4}\right)$. On the one hand,

$$
a\left(q_{4}\right)=t \rho_{1} t\left(q_{4}\right)=t \rho_{1}\left(q_{4} q_{3} q_{4}^{-1}\right)=t\left(q_{4} u q_{4}^{-1}\right)=q_{4} t(u) q_{4}^{-1},
$$

where $u=\rho_{1}\left(q_{3}\right)$ is a word in $q_{1}, q_{2}, q_{3}$. Thus $t(u)$, and also $a\left(q_{4}\right)$, are words in $q_{1}, q_{2}, q_{4}$. On the other hand,

$$
a\left(q_{4}\right)=\rho_{3}^{-1} t^{-1} \rho_{2}^{-1}\left(q_{4}\right)=\rho_{3}^{-1} t^{-1}\left(q_{4}\right)=\rho_{3}^{-1}\left(q_{3}\right)
$$

which is a word in $q_{1}, q_{2}, q_{3}$. It follows that $a\left(q_{4}\right)=\rho_{3}^{-1}\left(q_{3}\right)$ must lie in $\left\langle q_{1}, q_{2}\right\rangle$, and we are in the same situation as case (2) above.

Finally, suppose two of the $i_{j}$ have opposite signs. Permuting and inverting the relation as necessary, we may assume $i_{1}=1$ and $i_{2}=i_{3}=-1$. Set $\delta=$ rst. A simple computation shows that conjugation by $\delta$ takes $A_{R}$ to $A_{T}$. Let $\tau_{1}=$ $\delta \rho_{1} \delta^{-1} \in A_{T}$. Conjugating the two expressions for $a$ by $(r s)$ gives

$$
\begin{aligned}
(r s) t \rho_{1} t^{-1}(r s)^{-1} & =(r s) \rho_{3}^{-1} t \rho_{2}^{-1}(r s)^{-1} \\
\tau_{1} & =(r s) \rho_{3}^{-1} t \rho_{2}^{-1}(r s)^{-1} \\
\tau_{1}\left(r s \rho_{2}\right) & =\left(r s \rho_{3}^{-1}\right) t .
\end{aligned}
$$

Applying Lemma 4.1 with $\tau_{2}=t$ gives the desired result. This completes the proof of the lemma.

\section{The MAIN THEOREM}

We are now ready to prove that the spherical Deligne complex for the four strand braid group is CAT(1). By Theorem 2.1, we must verify that links of vertices in $\mathcal{D}^{0}$ are CAT(1) and that $\mathcal{D}^{0}$ has no closed geodesics of length less than $2 \pi$. The link of a vertex in $\mathcal{D}^{0}$ is the spherical Deligne complex of a 2-generator Artin group (namely the parabolic subgroup stabilizing that vertex) which is CAT(1) by [5], Proposition 4.4.5. Thus, it remains to show that $\mathcal{D}^{0}$ has no closed geodesics of length $<2 \pi$.

In [12, Elder and McCammond describe an algorithmic method for determining possible configurations for closed geodesics of length less than $2 \pi$ in a given, piecewise spherical 2-complex. Their method involves "developing" geodesic segments onto a 2-sphere. We use a similar method here, but in our case the process can be greatly simplified by developing geodesics onto $\mathcal{C}^{0}$, rather than onto an abstract 2 -sphere, and using the simplicial structure of $\mathcal{C}^{0}$. This will allow us to quickly reduce to the case of a geodesic edge path.

If a local geodesic $\gamma$ in $\mathcal{D}^{0}$ does not pass through any vertices, we can map the sequence of chambers containing $\gamma$ to a sequence of chambers in $\mathcal{C}^{0}$ such that the 




FiguRe 5. An $r$-cube in $\mathcal{C}^{0}$

image of $\gamma$ is again a local geodesic. This map is unique, once an initial chamber has been chosen. This process is called "developing" $\gamma$ onto $\mathcal{C}^{0}$. (Note that it is not the same as the image of $\gamma$ under the projection map $\mathcal{D}^{0} \rightarrow \mathcal{C}^{0}$.)

In general, we cannot develop a geodesic across a vertex. However, in the case of a vertex of type $\{r, t\}$, the developing map still works as we now describe. Let $K^{0}$ be the fundamental chamber of $\mathcal{C}^{0}$. Then the union of the chambers $K^{0}, r K^{0}, t K^{0}, r t K^{0}$ forms a quadrilateral $Q$ in $\mathcal{C}^{0}$ with all sides of type $s$ (see Figure (5). We call any $W$-translate of $Q$ an $r t$-cube of $\mathcal{C}^{0}$. Similarly, if $\hat{K}^{0}$ is the fundamental chamber of $\mathcal{D}^{0}$, then for any $i, j \neq 0$, the union of the chambers $\hat{K}^{0}, r^{i} \hat{K}^{0}, t^{j} \hat{K}^{0}, r^{i} t^{j} \hat{K}^{0}$ forms a quadrilateral $\hat{Q}$ in $\mathcal{D}^{0}$ with all sides of type $s$. We call any $A$-translate of such a quadrilateral an $r t$-cube in $\mathcal{D}^{0}$.

Given a (type preserving) projection of a chamber $a \hat{K}^{0}$ onto a chamber $w K^{0}$ of $\mathcal{C}^{0}$ and an $r$-cube $a \hat{Q}$, this projection extends to a unique isometry of $a \hat{Q}$ onto an $r t$-cube of $\mathcal{C}^{0}$. It follows that any geodesic segment in $a \hat{Q}$ can be developed to a geodesic segment in $\mathcal{C}^{0}$, even if it passes through the center vertex of the cube. Thus, for the purpose of developing local geodesics in $\mathcal{D}^{0}$ to local geodesics in $\mathcal{C}^{0}$, vertices of type $\{r, t\}$ can be treated as nonsingular points. We will sometimes refer to vertices of type $\{r, t\}$ as "nonsingular" vertices and those of type $\{r, s\}$ or $\{s, t\}$ as "singular" vertices. A pair of edges of type $t$ (respectively $r$ ) meeting at a nonsingular vertex forms the diagonal of some $r t$-cube and will be refered to as a $t$-diagonal (respectively $r$-diagonal).

Lemma 5.1. Let $v_{1}$ and $v_{2}$ be distinct vertices in $\mathcal{D}^{0}$ of type $\{r, t\}$. Then $\operatorname{Star}\left(v_{1}\right) \cap$ $\operatorname{Star}\left(v_{2}\right)$ is either empty, a single vertex, or a single edge. In particular, two distinct $t$-diagonals (or $r$-diagaonals) meet in at most one vertex.

Proof. It suffices to prove the case where $v_{1}=A_{\{r, t\}}$. We first show that $\operatorname{Star}\left(v_{1}\right) \cap$ $\operatorname{Star}\left(v_{2}\right)$ contains at most one vertex of type $T=\{s, t\}$ and at most one vertex of type $R=\{r, s\}$. Let $v_{2}=a A_{\{r, t\}}$ and suppose $v_{3}=b A_{T}$ lies in $\operatorname{Star}\left(v_{1}\right) \cap \operatorname{Star}\left(v_{2}\right)$. Then $b A_{T} \cap A_{\{r, t\}} \neq \emptyset$ so the coset representative $b$ can be chosen to be $r^{i}$ for some $i$. Since $b A_{T} \cap a A_{\{r, t\}}$ is also nonempty, $b A_{T}=r^{i} A_{T}$ contains an element of the form $a r^{j}$. Thus, $a r^{j}=r^{i} \tau_{1}$ for some $\tau_{1} \in A_{T}$.

Now if $v_{4}$ is another vertex of type $T$ lying in $\operatorname{Star}\left(v_{1}\right) \cap \operatorname{Star}\left(v_{2}\right)$, then we likewise have $v_{4}=r^{k} A_{T}$ and $a r^{l}=r^{k} \tau_{2}$. Thus, $a=r^{i} \tau_{1} r^{-j}=r^{k} \tau_{2} r^{-l}$, and hence 
$\tau_{1} r^{l-j}=r^{k-i} \tau_{2}$. By Lemma 4.1 either $l-j=k-i=0$, or $\tau_{1}=\tau_{2}=t^{n}$ for some $n$.

In the latter case, $a=r^{i-j} t^{n} \in A_{\{r, t\}}$, so $v_{1}=v_{2}$ which contradicts the assumption that $v_{1}$ and $v_{2}$ are distinct vertices. In the former case, we get $i=k$ so $v_{3}=v_{4}$, and we conclude that $\operatorname{Star}\left(v_{1}\right) \cap \operatorname{Star}\left(v_{2}\right)$ contains at most one vertex $r^{i} A_{T}$ of type $T$. By a completely analogous argument, it contains at most one vertex $t^{j} A_{R}$ of type $R$. Since $\mathcal{D}^{0}$ is a flag complex, if $\operatorname{Star}\left(v_{1}\right) \cap \operatorname{Star}\left(v_{2}\right)$ contains the vertices $r^{i} A_{T}$ and $t^{j} A_{R}$, then it must also contain the edge $r^{i} t^{j} A_{s}$ connecting them. We conclude that $\operatorname{Star}\left(v_{1}\right) \cap \operatorname{Star}\left(v_{2}\right)$ is either empty, or consists of a single vertex or a single edge.

We will need to analyze certain edge paths in $\mathcal{D}^{0}$. Suppose $e_{1}$ and $e_{2}$ are two edges meeting at a vertex $v$ of type $S_{1} \subset S$. Let $a \hat{K}^{0}$ be a chamber containing $e_{1}$ and $b \hat{K}^{0}$ a chamber containing $e_{2}$. Then $b=a \rho_{1}$ for some $\rho_{1} \in A_{S_{1}}$. Now suppose that $e_{1}, e_{2}, \ldots, e_{k}$ is a closed edge path in $\mathcal{D}^{0}$ and the vertex between $e_{i}$ and $e_{i+1}$ is of type $S_{i}$. Repeating the process above at each vertex, we get a relation $\rho_{1} \rho_{2} \cdots \rho_{k}=1$, where $\rho_{i} \in A_{S_{i}}$. Moreover, if $e_{i}, e_{i+1}$ forms an $r$-diagonal (respectively $t$-diagonal), then we can choose our chambers so that $\rho_{i} \in A_{t}$ (respectively $\rho_{i} \in A_{r}$ ).

Lemma 5.2. Suppose that $p$ is a closed edge path in $\mathcal{D}^{0}$ consisting of 4 edges of type $s$. Then $p$ is the boundary of a unique rt-cube in $\mathcal{D}^{0}$. In particular, $p$ is not locally geodesic at any of its four vertices.

Proof. Edges of type $s$ connect vertices of type $R=\{r, s\}$ to vertices of type $T=\{s, t\}$. It suffices to consider the case where the first edge of $p$ is the edge $A_{s}$ of the fundamental chamber. By the discussion above, $p$ corresponds to a relation $\tau_{1} \rho_{1} \tau_{2} \rho_{2}=1$, where $\rho_{i} \in A_{R}$ and $\tau_{i} \in A_{T}$. By Lemma 4.1 the edges of $p$ are then

$$
\begin{aligned}
& e_{1}=A_{s}=s^{k_{1}} A_{s}, \\
& e_{2}=\tau_{1} A_{s}=s^{k_{1}} t^{i} A_{s}, \\
& e_{3}=\tau_{1} \rho_{1} A_{s}=s^{k_{1}} t^{i} r^{j} A_{s}, \\
& e_{4}=\tau_{1} \rho_{1} \tau_{2} A_{s}=s^{k_{1}} r^{j} A_{s} .
\end{aligned}
$$

Thus, $p$ is the boundary of an $r t$-cube $s^{k_{1}} \hat{Q}$. The uniqueness of this cube follows from Lemma 5.1.

Lemma 5.3. Suppose $p$ is a triangle in $\mathcal{D}^{0}$ consisting of three $r$-diagonals (respectively t-diagonals). Then $p$ is not locally geodesic at any of its three (singular) vertices.

Proof. By the discussion above, $p$ corresponds to a relation of the form

$$
t^{i_{1}} \rho_{1} t^{i_{2}} \rho_{2} t^{i_{3}} \rho_{3}=1
$$

where two edges of $p$ meeting at a singular vertex are of the form $e=a A_{r}$ and $e^{\prime}=a \rho_{j} A_{r}$ for some $a$. By Lemma 4.2, we have $\rho_{j}=r^{n} s^{k} r^{m}$ for some $n, k, m$. Thus we have $e^{\prime}=a r^{n} s^{k} A_{r}$ and $e=a r^{n} A_{r}$, so the angle between $e^{\prime}$ and $e$ is the same as the angle between $s^{k} A_{r}$ and $A_{r}$, namely $2 \pi / 3$.

Theorem 5.4. The spherical Deligne complex $\mathcal{D}^{0}$ for the braid group on four strands is $C A T(1)$. 

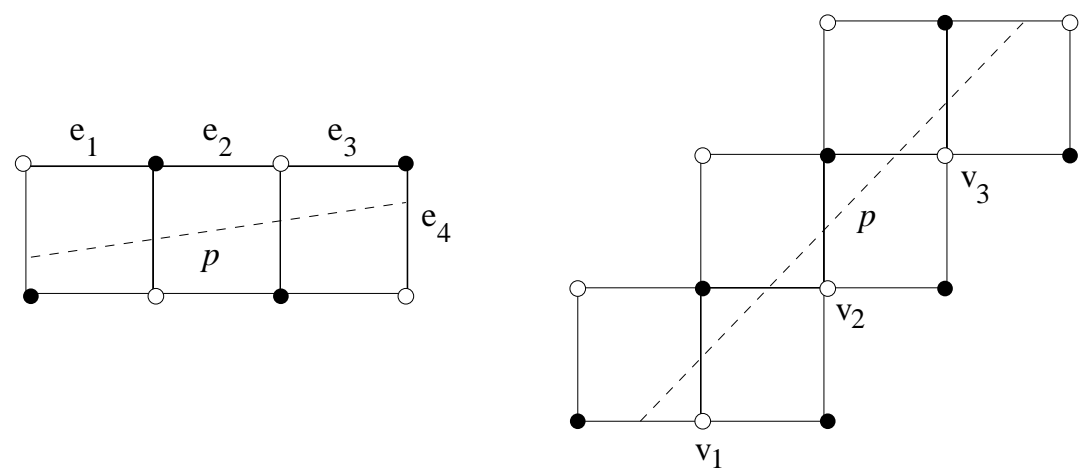

FIGURE 6. Geodesics with no singular vertices

Remark. Since $\mathcal{D}^{0}$ is the link of the cone point in $\mathcal{D}$ with respect to the Moussong metric, this theorem is equivalent to the statement that the Moussong metric on the Deligne complex for the 4-strand braid group is $\mathrm{CAT}(0)$.

Proof. We must show that $\mathcal{D}^{0}$ has no closed geodesics of length $<2 \pi$. First consider the case of a geodesic $p$ which passes through no vertices of type $R$ or $T$. Then $p$ can be developed onto a local geodesic $q$ in $\mathcal{C}^{0}$ which also contains no vertices of type $R$ or $T$. Note, however, that since $p$ has length less than $2 \pi, q$ will not be a closed geodesic in $\mathcal{C}^{0}$. It is easy to see (Figure 5) that any local geodesic in $\mathcal{C}^{0}$ which crosses an $r t$-cube from the interior of one edge to the interior of the opposite edge must do the same in the adjacent $r t$-cube and the distance across these two $r t$-cubes is $\pi$. Thus, $q$ is one of two types: either it crosses every $r t$-cube from one side to the opposite side, or it crosses every $r t$-cube from one side to an adjacent side.

In the first case, it suffices to show that $p$ (and hence $q$ ) crosses four $r t$-cubes (since this forces it to have length $2 \pi$ ). Lemma 5.1 rules out the possibility that $p$ closes up after crossing only 2 cubes. If it closes up after crossing three, then the three cubes shown on the left in Figure 6 form a möbius strip in $\mathcal{D}^{0}$ (since different colors represent different types of vertices). In this case, the edge path $e_{1}, e_{2}, e_{3}, e_{4}$ indicated in the figure forms a quadrilateral of type $s$-edges, hence bounds an $r t$-cube. This is impossible by Lemma 5.1

In the case where $q$ cuts across a corner of each $r t$-cube, it must cross six cubes to have length $2 \pi$. (This can be seen by looking at any great circle in $\mathcal{C}^{0}$ which cuts across the corner of an $r t$-cube.) The $r t$-cubes in $\mathcal{D}^{0}$ containing $p$ are pairwise joined along edges as shown on the right in Figure 6. In order for $p$ to close up after crossing less than six corners, two of the white vertices $v_{1}, v_{2}, v_{3}$ would have to be identified. But this contradicts Lemma 5.1, so $p$ must traverse all six $r t$-cubes which makes $q$ a full great circle in $\mathcal{C}^{0}$. This completes the case in which $p$ contains no vertices of type $R$ or $T$.

At the other extreme, suppose $p$ is a geodesic edge path. Then every edge of type $t$ or $r$ in $p$ must be followed by another of the same type. Thus $p$ consists of $t$-diagonals, $r$-diagonals, and edges of type $s$. By abuse of terminology, we will refer to all three of these as "edges". By Lemma 5.1, we may assume that $p$ consists of 

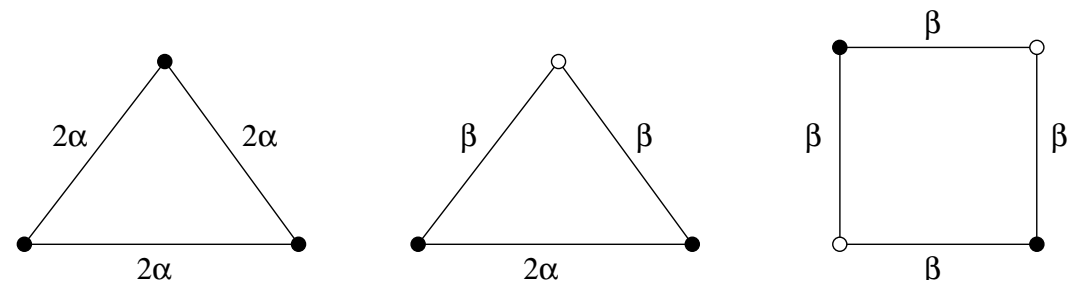

Figure 7. Possible closed edgepaths $p$

at least three such edges. As in Lemma 3.1, we let

$$
\begin{aligned}
2 \alpha & =\text { length of an } r \text {-diagonal or } t \text {-diagonal, } \\
\beta & =\text { length of an edge of type } s .
\end{aligned}
$$

Consider the possible configurations of edges of length $2 \alpha$ and $\beta$ which make up $p$. By Lemma $3.12 \alpha>\beta>\alpha$ and $2 \alpha+\beta=\pi$. In particular, $6 \beta>4 \alpha+2 \beta=2 \pi$, and $8 \alpha>2 \alpha^{\prime}+2 \beta=2 \pi$. Taking into account that edges of length $2 \alpha$ connect vertices of the same type, while edges of length $\beta$ connect vertices of different types, a simple exercise shows that the only possible configurations for $p$ are the three in Figure 7

By Lemmas 5.3 and 5.2 the triangle with all side lengths $2 \alpha$ and the quadrilateral with all side lengths $\beta$ fail to be locally geodesic at every vertex. That leaves the triangle $\Delta$ with side lengths $2 \alpha, \beta, \beta$. Suppose that the edge $e$ of length $2 \alpha$ is a $t$-diagonal. (The $r$-diagonal case is entirely analogous.) Then $\Delta$ can be translated so that $e$ is the pair of edges $A_{t}, r^{j} A_{t}$ for some $j$. Left multiplying by $t$, we get a new triangle, $t \Delta$, which is also of type $2 \alpha, \beta, \beta$ and still contains $e$ as its $2 \alpha$ edge. Together, $\Delta$ and $t \Delta$ give rise to a quadrilateral with all sides of type $s$. By Lemma 5.2 this quadrilateral bounds an $r t$-cube which, by Lemma 5.1, must contain $e$ as its $t$-diagonal. In other word, $\Delta$ bounds half of an $r t$-cube, so the angles at the vertices of $\Delta$ are $\pi / 3, \pi / 3$, and $2 \pi / 3$. We have, in fact, shown that a closed edge path of length less than $2 \pi$ fails to be geodesic at every singular vertex.

Finally, suppose $p$ is a closed geodesic in $\mathcal{D}^{0}$ of length less than $2 \pi$ which is not an edge path, but contains at least one singular vertex. Divide $p$ into segments $p=p_{1} p_{2} \ldots p_{n}$ such that each $p_{i}$ begins and ends at a singular vertex but contains no such vertices in its interior. Developing $p_{i}$ onto $\mathcal{C}^{0}$, we get a locally geodesic segment $q_{i}$ connecting two vertices in $\mathcal{C}^{0}$ of type $T$ or $R$. Since the point antipodal to any singular vertex in $\mathcal{C}^{0}$ is another singular vertex (of the opposite type), each $q_{i}$ is either an edge or half of a great circle. Since length $(q) \leq 2 \pi$, exactly one $q_{i}$ is a half circle, say $q_{1}$. This half circle can be rotated, with endpoints fixed, until it becomes an edge path with one vertex of type $T$ or $R$ in its interior. This rotation lifts to $\mathcal{D}^{0}$, to give a geodesic edgepath $p_{1}^{\prime}$ with the same endpoints and same length as $p_{1}$. The resulting path $p^{\prime}=p_{1}^{\prime} p_{2} \ldots p_{n}$ is a closed edge path of length less than $2 \pi$. By the discussion above, $p^{\prime}$ fails to be locally geodesic at every singular vertex. But this is a contradiction since $p_{1}^{\prime}$ is necessarily geodesic at its interior vertex.

Recall that $\mathrm{Sym}_{4}$ denotes the symmetric group on 4 letters. Combining the results of [5] with the theorem above we obtain the following corollary.

Corollary 5.5. Let $(W, S)$ be a Coxeter system such that every finite, irreducible special subgroup $W_{T}$ is either $S_{y m_{4}}, \mathbb{Z}_{2}$, or a dihedral group, and let $A$ be the 
associated Artin group. Then the Deligne complex $\mathcal{D}_{A}$ is $C A T(0)$, and the orbit space $\mathcal{H}_{W} / W$ of the associated hyperplane complement is a $K(A, 1)$-space.

Proof. For $T \in \mathcal{S}^{f}$, the decomposition of $\left(W_{T}, T\right)$ into irreducible factors gives a decomposition of $\left(A_{T}, T\right)$ into a direct product of copies of $\mathbb{Z}, 2$-generator Artin groups, and 4-strand braid groups. It follows (cf. [3], Thm. 3.1) that the link of the vertex $A_{T}$ in $\mathcal{D}_{A}$ decomposes as a join of spherical Deligne complexes for the factors. The spherical Deligne complex for a 2-generator Artin group is CAT(1) by [5], Proposition 4.4.5, and the spherical Deligne complex for the 4-strand braid group is CAT(1) by Theorem 5.4

In particular, we have

Corollary 5.6. Let $(A, S)$ be an Artin system whose Deligne complex $\mathcal{D}_{A}$ has dimension $\leq 3$. If $m(s, t) \neq 4,5$ for all $s, t \in S$, then $\mathcal{D}_{A}$ is $C A T(0)$ and the orbit space $\mathcal{H}_{W} / W$ of the associated hyperplane complement is a $K(A, 1)$-space.

The primary motivation for this paper was the $K(\pi, 1)$ conjecture for hyperplane complements. In closing, however, we note that there are other, algebraic consequences of having a CAT(0) Deligne complex. For example, in [16, Salvetti proves that there is a finite cell complex of the same dimension as $\mathcal{D}_{A}$ which is homotopy equivalent to the space $\mathcal{H}_{W} / W$ (see also [4]). Combining this with Corollary 5.5] we get

Corollary 5.7. Let $A$ be as in Corollary 5.5. Then $A$ has a finite $K(\pi, 1)$-space of dimension $n=\max \left\{|T| \mid T \in \mathcal{S}^{f}\right\}$. In particular, $A$ is torsion-free and has cohomological dimension $n$.

In his thesis [13, Godelle determines the normalizer, commensurator, and quasicentralizer of any finite parabolic subgroup of $A$ under the assumption that $\mathcal{D}_{A}$ is CAT(0). In [9, under the same hypothesis, Crisp proves that if $G$ is a group of symmetries of an Artin system $(A, S)$, then the subgroup of $A$ fixed by $G$ is itself an (explicitly described) Artin group. These results can now be applied to the Artin groups in Corollary 5.5.

\section{REFERENCES}

[1] M. Bridson and A. Haefliger. Metric Spaces of Non-positive Curvature. Springer-Verlag, Berlin, 1999. MR 2000k:53038

[2] R. Charney. Injectivity of the positive monoid for some infinite type Artin groups. In J. Cossey, C. F. Miller, W. D. Neumann, and M. Shapiro, editors, Geometric Group Theory Down Under, pages 103-118, Berlin, 1999. Walter de Gruyter. MR 2000h:20073

[3] R. Charney. The Tits conjecture for locally reducible Artin groups. International Journal of Algebra and Computation, 10:783-797, 2000. MR 2002d:20057

[4] R. Charney and M. W. Davis. Finite $K(\pi, 1)$ 's for Artin groups. In F. Quinn, editor, Prospects in Topology, number 138 in Annals of Math Studies, pages 110-124. Princeton University Press, 1995. MR 97a:57001

[5] R. Charney and M. W. Davis. The $K(\pi, 1)$-problem for hyperplane complements associated to infinite reflection groups. Journal of the American Mathematical Society, 8(3):597-627, 1995. MR 95i:52011

[6] R. Charney and A. Lytchak. Metric characterizations of spherical and Euclidean buildings. Geometry and Topology, 5:521-550, 2001. MR 2002h:51008

[7] R. Charney and D. Peifer. The $K(\pi, 1)$-conjecture for the affine braid groups. to appear in Commentarii Mathematici Helvetici. 
[8] J. Crisp. Injective maps between Artin groups. In J. Cossey, C. F. Miller, W. D. Neumann, and M. Shapiro, editors, Geometric Group Theory Down Under, pages 119-137, Berlin, 1999. Walter de Gruyter. MR 2001b:20064

[9] J. Crisp. Symmetrical subgroups of Artin groups. Advances in Mathematics, 152:159-177, 2000. MR 2001c:20083

[10] M. W. Davis. Groups generated by reflections and aspherical manifolds not covered by Euclidean space. Annals of Mathematics, 117:293-324, 1983. MR 86d:57025

[11] P. Deligne. Les immeubles des groupes de tresses généralises. Inventiones Math., 17:273-302, 1972. MR 54:10659

[12] M. Elder and J. McCammond. Curvature testing in 3-dimensional metric polyhedral complexes. Experimental Mathematics, 11:143-158, 2002.

[13] E. Godelle. Normalisateurs et centralisateurs des sous-groupes paraboliques dans les groups d'Artin-Tits. Ph.D. thesis, Université de Picardie Jules Verne, 2001.

[14] M. Gromov. Hyperbolic groups. In Essays in Group Theory, number 8 in Math. Sci. Res. Inst. Publ., pages 75-264. Springer-Verlag, New York, 1987. MR 89e:20070

[15] G. Moussong. Hyperbolic Coxeter groups. Ph.D. thesis, Ohio State University, 1988.

[16] M. Salvetti. Topology of the complement of real hyperplanes in $\mathbb{C}^{n}$. Inventiones Mathematica, 88:603-618, 1987. MR 88k:32038

[17] H. van der Lek. Extended Artin groups. Proceedings of the Symposium in Pure Mathematics, 40:117-121, 1983. MR 85b:14005

[18] E. B. Vinberg. Discrete linear groups generated by reflections. Math. USSR Izvestija, 5(5):1083-1119, 1971. MR 46:1922

Department of Mathematics, The Ohio State University, 231 W. 18th Ave, Columbus, Оніо 43210

E-mail address: charney@math.ohio-state.edu

Current address: Department of Mathematics, Brandeis University, Waltham, Massachusetts 02254

E-mail address: charney@brandeis.edu 